\title{
Article \\ Regularization Algorithms for Linear Copositive Programming Problems: An Approach Based on the Concept of Immobile Indices
}

\author{
Olga Kostyukova ${ }^{1,+} \mathbb{D}$ and Tatiana Tchemisova ${ }^{2, *,+}$ \\ 1 Institute of Mathematics, National Academy of Sciences of Belarus, Surganov Str. 11, 220072 Minsk, Belarus; \\ kostyukova@im.bas-net.by \\ 2 Department of Mathematics, Campus Universitário Santiago, University of Aveiro, 3810-193 Aveiro, Portugal \\ * Correspondence: tatiana@ua.pt \\ + These authors contributed equally to this work.
}

Citation: Kostyukova, O.;

Tchemisova, T. Regularization

Algorithms for Linear Copositive

Programming Problems: An

Approach Based on the Concept of Immobile Indices. Algorithms 2022, 15, 59. https://doi.org/10.3390/ a15020059

Academic Editor: Frank Werner

Received: 24 January 2022

Accepted: 7 February 2022

Published: 11 February 2022

Publisher's Note: MDPI stays neutral with regard to jurisdictional claims in published maps and institutional affiliations.

Copyright: (C) 2022 by the authors. Licensee MDPI, Basel, Switzerland. This article is an open access article distributed under the terms and conditions of the Creative Commons Attribution (CC BY) license (https:// creativecommons.org/licenses/by/ $4.0 /)$.

\begin{abstract}
In this paper, we continue an earlier study of the regularization procedures of linear copositive problems and present new algorithms that can be considered as modifications of the algorithm described in our previous publication, which is based on the concept of immobile indices. The main steps of the regularization algorithms proposed in this paper are explicitly described and interpreted from the point of view of the facial geometry of the cone of copositive matrices. The results of the paper provide a deeper understanding of the structure of feasible sets of copositive problems and can be useful for developing a duality theory for these problems.
\end{abstract}

Keywords: linear copositive programming; normalized immobile index set; regularization algorithm; minimal cone; facial reduction

\section{Introduction}

The problems of copositive programming (CoP) form a special class of conic optimization problems in which the images of feasible sets belong to a convex cone of the so-called copositive matrices (i.e., matrices that are positive semidefinite on the non-negative orthant). Copositive models exist for many important applications, including $\mathcal{N} \mathcal{P}$-hard problems. For references on the motivations and applications of CoP, see, e.g., [1-4].

In linear $\mathrm{CoP}$, the objective function is linear and the constraints are formulated using linear matrix functions. Linear copositive problems are closely related to linear semi-infinite programming (SIP) and semidefinite programming (SDP). Although copositive and semidefinite problems are special cases of SIP problems, CoP deals with more complex and less studied problems than SDP. There are many publications devoted to the theory and methods of SIP, CoP, and SDP. We refer interested readers to [1,3,5-7], and the references therein.

In convex and conic optimization, optimality conditions and duality results are usually formulated when certain regularity conditions, the so-called constraint qualifications (CQ), are met (see, for example, [6-8]). Such conditions should guarantee the fulfillment of Karush-Kuhn-Tucker (KKT)-type optimality conditions and the property of strong duality which consists of the fact that when the primal problem and the corresponding Lagrangian dual problem are consistent, the optimal values of these problems are equal and the dual problem reaches its maximum. Strong duality is the cornerstone of convex optimization and plays a particularly important role in the stability of numerical methods. One of the most used CoP regularity conditions is the Slater CQ. The most efficient and popular SIP and SDP software is based either on interior point methods or on the discretization approach and the assumption that the Slater regularity condition is satisfied.

Unfortunately, even for linear copositive problems, the regularity conditions cannot always be guaranteed. In the cases where regularity conditions are not met, there is no 
guarantee that an optimal solution satisfies the KKT-type optimality conditions, the firstorder optimality conditions of the Fritz-John-type become degenerate (they are satisfied for all feasible solutions and hence are not informative), and the strong duality relation may fail. This creates numerical difficulties in solving problems.

As a rule, the violation of a CQ is caused by an inconvenient description of the feasible set of the optimization problem. Thus, the idea of regularization arises quite naturally. Regularization aims to improve the modeling of an optimization problem by obtaining an equivalent and more convenient reformulation of this problem with some given regularity properties.

In the literature there are several approaches to the regularization of conic optimization problems. In $[9,10]$, the concept of the minimal cone of constraints was used by Borwein and Wolkowicz to regularize abstract convex and conic convex problems for which any CQ fails. The algorithm proposed there to describe the minimal cone is based on the gradual reduction of the faces of the constraints' cone, and the authors called it the facial reduction algorithm (FRA). Another approach, dual regularization, was proposed by Luo, Sturm, and Zhang (see [11] and the references therein). This approach aims to reduce the duality gap (the differences between the primal and dual optimal values) of regularized problems by expanding the dual constraints' cone. On iteration of the FRA proposed by Waki and Muramatsu in [12], the faces of a given conic problem are transformed in such a way that each reduced face is dual to the cone generated in the corresponding dual regularization step from [11].

The facial reduction approach has been successfully applied to SDP and secondorder cone programming problems, and to certain classes of optimization problem over symmetric (i.e., self-dual and homogeneous) and nice cones (see, for example, [13-16]). At the same time, the question of the effectiveness of such an approach to other classes of problems remains open. This is because the known FRAs are more conceptual than practical.

In [17], using our previous results published in [18,19], and others, we proposed a different approach to the regularization of linear $\mathrm{CoP}$ problems. This approach is based on the concept of immobile indices (i.e., indices of the constraints that are active for all feasible solutions) originally introduced for SIP and SDP problems. We used this approach to develop two finite algorithms for the regularization of linear CoP problems: one method is called one-step regularization and the another is the iterative algorithm REG-LCoP (regularization of linear copositive problems). The first method consists of one step, but certain difficulties may arise when applying it, since it is based on a priori possession of some additional information about some finite subset of immobile indices. Unlike the one-step regularization, the algorithm REG-LCoP does not require any additional information in advance. Comparing this algorithm with other algorithms, we showed that it is more constructive because its iterations (using the concept of immobile indices) can be more explicitly described than the iterations of other FRA-based algorithms (which use the concept of the minimal cone).

As far as we know, the algorithm REG-LCoP is the first constructive regularization procedure developed specifically for linear copositive problems. This algorithm is important not only because it is based on previously unexplored properties of copositive problems, but also conceptually, since new algorithms and methods can be developed on its basis.

In this paper, we propose three new algorithms that are modifications of REG-LCoP. Although these algorithms have structures similar to that of the REG-LCoP algorithm, they do not require either the feasibility of the original optimization problem or the initial knowledge of a set of immobile indices and can be considered as developments in the approach to the regularization of copositive problems, started in [17].

All the algorithms presented in the paper use the properties of the copositive problem established here and are based on alternative representations of its feasible set that are obtained using the concept of normalized immobile indices. The algorithms are finite and their iterations are explicitly described. 
The main differences between the modifications of the REG-LCoP algorithm presented here consist in the auxiliary problems that are solved in their iterations and in the properties of their solution sets. According to their specific needs, a user can choose the most appropriate version of the main algorithm.

It is important to emphasize that the algorithms described in the paper are important not only from the point of view of applications, but also as they contribute to the theoretical study of the structural properties of copositive problems. The main steps of the algorithms are interpreted in the paper from the point of view of the facial geometry of the cone of copositive matrices. Therefore, the results of the paper allow a deeper understanding of the structure of the feasible set of a given copositive problem and can serve as a basis for new types of optimality conditions and various forms of dual problems that satisfy strong duality relations.

The paper is organized as follows. Section 1 hosts the Introduction. In Section 2, we formulate the problem, and introduce basic notation and some preliminary results. The properties of the set of normalized immobile indices for the copositive problem under investigation are considered in Section 3. In Section 4, we describe and justify three versions of the REG-LCoP algorithm from [17]. A comparison of these methods among each other and with one method from the literature is provided. Section 5 contains conclusions, and some technical proofs are given in the Appendix A.

\section{Problem Statement, Basic Notation, and Some Preliminary Results}

Given an integer $p>1$, denote by $\mathbb{R}_{+}^{p}$ the set of all $p$ vectors with non-negative components (the non-negative orthant in $\mathbb{R}^{p}$ ); let $\mathcal{S}^{p}$ and $\mathcal{S}_{+}^{p}$ be the space of real symmetric $p \times p$ matrices and the cone of symmetric positive semidefinite $p \times p$ matrices, respectively; and let $\mathcal{C O P}{ }^{p}$ stay for the cone of symmetric copositive $p \times p$ matrices:

$$
\mathcal{C O P} \mathcal{P}^{p}:=\left\{D \in \mathcal{S}^{p}: t^{\top} D t \geq 0 \forall t \in \mathbb{R}_{+}^{p}\right\} .
$$

Consider a linear copositive programming problem

$$
\min _{x \in \mathbb{R}^{n}} c^{\top} x, \quad \text { s.t. } \mathcal{A}(x) \in \mathcal{C O} \mathcal{P}^{p},
$$

where $x=\left(x_{1}, \ldots, x_{n}\right)^{\top}$ is the vector of decision variables. The data of the problem above consist of the vector $c \in \mathbb{R}^{n}$ and the constraints matrix function $\mathcal{A}(x)$ in the form

$$
\mathcal{A}(x):=\sum_{j=1}^{n} A_{j} x_{j}+A_{0}
$$

with given matrices $A_{j} \in \mathcal{S}^{p}, j=0,1, \ldots, n$.

It is well known (see, e.g., [5]) that the copositive problem (1) is equivalent to the following convex SIP problem:

$$
\min _{x \in \mathbb{R}^{n}} c^{\top} x, \text { s.t. } t^{\top} \mathcal{A}(x) t \geq 0 \quad \forall t \in T,
$$

with a $p$-dimensional compact index set $T$. We define this set as a simplex

$$
T:=\left\{t \in \mathbb{R}_{+}^{p}: \mathbf{e}^{\top} t=1\right\},
$$

where $\mathbf{e}=(1,1, \ldots, 1)^{\top} \in \mathbb{R}^{p}$.

Denote by $X$ the feasible set of the equivalent problems (1) and (3):

$$
X:=\left\{x \in \mathbb{R}^{n}: \mathcal{A}(x) \in \mathcal{C O} \mathcal{P}^{p}\right\}=\left\{x \in \mathbb{R}^{n}: t^{\top} \mathcal{A}(x) t \geq 0 \quad \forall t \in T\right\} .
$$

Evidently, either the set $X$ is convex or it is empty. 
Let us reformulate some standard definitions for the cone $\mathcal{C O P} p$. A convex subset $\mathbf{F}$ of the cone $\mathcal{C O P} \mathcal{P}^{p}$ is its face if for any $x \in \mathcal{C O} \mathcal{P}^{p}$ and $y \in \mathcal{C O P}{ }^{p}$, the inclusion $x+y \in \mathbf{F}$ implies $x \in \mathbf{F}, y \in \mathbf{F}$. Notice that any face of a cone is also a cone. A face $\mathcal{F}$ of $\mathcal{C O} \mathcal{P}^{p}$ is called exposed if it can be presented as the intersection of $\mathcal{C O P}^{p}$ with a supporting hyperplane.

Given the copositive problem $\mathcal{C O P}{ }^{p}$, let $\mathbf{F}_{\text {min }}$ be the smallest (by inclusion) face of $\mathcal{C O} \mathcal{P}^{p}$ containing the image set $\mathcal{D}$ of the constraints of this problem defined as follows:

$$
\mathcal{D}:=\{\mathcal{A}(x), x \in X\} .
$$

The face $\mathbf{F}_{\text {min }}$ will be called here the minimal face of the optimization problem (1).

According to the commonly used definitions, the constraints of the copositive problem (1) satisfy the Slater condition if

$$
\exists \bar{x} \in \mathbb{R}^{n} \text { such that } \mathcal{A}(\bar{x}) \in \operatorname{int} \mathcal{C O} \mathcal{P}^{p}=\left\{D \in \mathcal{S}^{p}: t^{\top} D t>0 \forall t \in \mathbb{R}_{+}^{p}, t \neq \mathbf{0}\right\},
$$

where int $\mathcal{C}$ stays for the interior of a set $\mathcal{C}$ and $\mathbf{0}$ denotes the null vector in the corresponding finite dimensional space.

As it was mentioned in the Introduction, in [17] we described a regularization algorithm REG-LCoP specially designed for problems in the form (1). In this paper we go further and develop new modifications of the algorithm REG-LCoP that are more explicit and have less additional requirements. To justify these modified algorithms, we need some preliminary results.

In what follows, to simplify notation, for $y=\left(y_{1}, \ldots, y_{n}\right)^{\top} \in \mathbb{R}^{n}, y_{0} \in \mathbb{R}$, and $\mu \in \mathbb{R}$, the vector $\left(y^{\top}, y_{0}\right)^{\top} \in \mathbb{R}^{n+1}$ will be denoted as $\left(y, y_{0}\right) \in \mathbb{R}^{n+1}$, and the vector $\left(y^{\top}, y_{0}, \mu\right)^{\top} \in$ $\mathbb{R}^{n+2}$ as $\left(y, y_{0}, \mu\right) \in \mathbb{R}^{n+2}$.

Introduce a matrix function $\mathcal{B}\left(y, y_{0}\right):=\sum_{j=1}^{n} A_{j} y_{j}+A_{0} y_{0}$ and consider the set

$$
\begin{aligned}
\mathcal{Z} & :=\left\{\left(y, y_{0}\right) \in \mathbb{R}^{n+1}: \mathcal{B}\left(y, y_{0}\right) \in \mathcal{C O} \mathcal{P}^{p}, y_{0} \geq 0\right\} \\
& =\left\{\left(y, y_{0}\right) \in \mathbb{R}^{n+1}: t^{\top} \mathcal{B}\left(y_{1} y_{0}\right) t \geq 0 \forall t \in T, y_{0} \geq 0\right\}
\end{aligned}
$$

Notice that $\mathcal{Z} \neq \varnothing$.

Given the sets $X \neq \varnothing$ and $\mathcal{Z}$ defined in (5) and (8), respectively, following [19], we define here the corresponding sets of (normalized) immobile indices $T_{i m}(X)$ and $T_{i m}(\mathcal{Z})$ :

$$
\begin{aligned}
T_{i m}(X) & :=\left\{t \in T: t^{\top} \mathcal{A}(x) t=0 \quad \forall x \in X\right\}, \\
T_{i m}(\mathcal{Z}) & :=\left\{t \in T: t^{\top} \mathcal{B}\left(y, y_{0}\right) t=0 \quad \forall\left(y, y_{0}\right) \in \mathcal{Z}\right\} .
\end{aligned}
$$

Generally speaking, the set of immobile indices of a given optimization problem is defined by the indices of constraints which are active for all feasible solutions.

Based on Lemma 1 and Proposition 1 in [19], let us formulate the following lemma.

Lemma 1. Given the linear copositive problem (1), suppose that $X \neq \varnothing$. Then

(i) the Slater condition (7) is equivalent to the emptiness of the set of normalized immobile indices $T_{\text {im }}(X)$,

(ii) The set $T_{i m}(X)$ is either empty or can be presented as a union of a finite number of convex closed bounded polyhedra.

For a vector $t=\left(t_{k}, k \in P\right)^{\top} \in \mathbb{R}_{+}^{p}$ with $P:=\{1,2, \ldots, p\}$, define the index set

$$
P_{+}(t):=\left\{k \in P: t_{k}>0\right\} .
$$


Given a set $\mathcal{C} \subset \mathbb{R}^{p}$ and a vector $l \in \mathbb{R}^{p}$, denote by $\rho(l, \mathcal{C})$ the distance between this set and the vector, $\rho(l, \mathcal{C}):=\min _{\tau \in \mathcal{C}} \sum_{k \in P}\left|l_{k}-\tau_{k}\right|$, and by conv $\mathcal{C}$, the convex hull of the set $\mathcal{C}$. Here and in what follows, for a vector $\tau \in \mathbb{R}^{p}$, we denote by $\tau_{k}, k \in P$, its components.

Consider a finite non-empty subset $W$ of the simplex $T$ defined in (4). Without loss of generality, we suppose that the elements of the set $W$ are indexed as follows:

$$
W=\{\tau(i), i \in I\} \subset T, 0<|I|<\infty,
$$

where $I$ is an index set. For the set $W$, define the number

$$
\sigma(W):=\min \left\{\tau_{k}(i), \quad k \in P_{+}(\tau(i)), i \in I\right\}>0,
$$

and the sets

$$
\begin{aligned}
\Omega(W) & :=\{t \in T: \rho(t, \mathrm{conv} W) \geq \sigma(W)\}, \\
\mathcal{X}(W):=\left\{x \in \mathbb{R}^{n}: \mathcal{A}(x) \tau(i) \geq 0 \forall i \in I ; t^{\top} \mathcal{A}(x) t \geq 0 \forall t \in \Omega(W)\right\}, & \mathcal{Z}(W):=\left\{\left(y, y_{0}\right) \in \mathbb{R}^{n+1}: \mathcal{B}\left(y, y_{0}\right) \tau(i) \geq 0 \forall i \in I ;\right. \\
& \left.t^{\top} \mathcal{B}\left(y, y_{0}\right) t \geq 0 \forall t \in \Omega(W), y_{0} \geq 0\right\} .
\end{aligned}
$$

Suppose that the constraints of problem (1) do not satisfy the Slater condition; i.e., the set of normalized immobile indices $T_{i m}(X)$ of this problem is not empty.

By following the proof of Theorem 5 in [18], we can prove the next theorem.

Theorem 1. Consider problem (1) with the feasible set $X \neq \varnothing$ given in (5) and the set $\mathcal{Z}$ given in (8). Then

(i) For any set $W \subset T_{i m}(X)$ in the form (11), the equality $X=\mathcal{X}(W)$ holds true, where the set $\mathcal{X}(W)$ is defined in (14).

(ii) For any set $W \subset T_{\text {im }}(\mathcal{Z})$ in the form (11), the equality $\mathcal{Z}=\mathcal{Z}(W)$ holds true, where the set $\mathcal{Z}(W)$ is defined in (15).

This theorem gives us alternative representations of the related sets $X$ and $\mathcal{Z}$ on the basis of a given finite set (11). This theorem and the results of the next section form a basis for new regularization algorithms that will be described below. Each of these algorithms successively generates a finite sequence of finite sets $W_{m}=\left\{\tau(i), i \in I_{m}\right\} \subset T$, $m=1, \ldots, m_{*}$, and hence the corresponding representations $\mathcal{Z}\left(W_{m}\right)$ of the non-empty set $\mathcal{Z}$. At the final iteration of the algorithm, a set $W_{m_{*}}$ is constructed which allows one either to recognize the infeasibility of the problem (1) or to obtain an equivalent representation $X\left(W_{m_{*}}\right)$ of the feasible set $X$ satisfying the regularity condition.

\section{Properties of the Set of Normalized Immobile Indices}

In this section, we consider a copositive problem in the form (1) with the corresponding sets $X, \mathcal{Z}, T_{i m}(X)$, and $T_{i m}(\mathcal{Z})$ defined in (5) and (8)-(10), respectively. The propositions below describe the properties of these sets, show the relations between the sets, and allow one to recognize the infeasibility of the problem (1). These results are then used in the next section to construct the algorithms for regularization of the CoP problem without a feasibility assumption.

Proposition 1. If $T_{\text {im }}(\mathcal{Z})=\varnothing$, then $X \neq \varnothing$ and $T_{\text {im }}(X)=\varnothing$.

Proof. Note that the set $\mathcal{Z}$ defined in (8) is not empty. Since $T_{i m}(\mathcal{Z})=\varnothing$, there exist a vector $\left(\tilde{y}, \tilde{y}_{0}\right) \in \mathcal{Z}$ and a number $\mu$ such that

$$
t^{\top} \mathcal{B}\left(\tilde{y}, \tilde{y}_{0}\right) t \geq \mu>0 \forall t \in T .
$$


If $\tilde{y}_{0}>0$, consider vector $\bar{x}:=\tilde{y} / \tilde{y}_{0}$. From (16), it follows that $t^{\top} \mathcal{A}(\bar{x}) t \geq \mu / \tilde{y}_{0}>0$ for all $t \in T$. Hence, $X \neq \varnothing$ and $T_{i m}(X)=\varnothing$.

Suppose now that $\tilde{y}_{0}=0$. Then relations (16) take the form

$$
t^{\top}\left(\sum_{j=1}^{n} A_{j} \tilde{y}_{j}\right) t \geq \mu>0 \forall t \in T
$$

Set: $\alpha_{0}:=\min _{t \in T} t^{\top} A_{0} t$ and $\theta:=\left\{\begin{array}{ll}1 & \text { if } \alpha_{0} \geq 0, \\ -2 \alpha_{0} / \mu & \text { if } \alpha_{0}<0,\end{array}\right.$ and consider the vector $\bar{x}:=\theta \tilde{y}$. Calculate

$$
\min _{t \in T} t^{\top} \mathcal{A}(\bar{x}) t \geq \theta \min _{t \in T} t^{\top}\left(\sum_{j=1}^{n} A_{j} \tilde{y}_{j}\right) t+\alpha_{0} \geq \theta \mu+\alpha_{0}= \begin{cases}\mu+\alpha_{0}>0, & \text { if } \alpha_{0} \geq 0 \\ -\alpha_{0}>0, & \text { if } \alpha_{0}<0 .\end{cases}
$$

This implies that $\bar{x} \in X \neq \varnothing$ and $T_{i m}(X)=\varnothing$. The proposition is proved.

Proposition 2. If $X \neq \varnothing$, then $T_{i m}(X)=T_{i m}(\mathcal{Z})$.

Proof. Let us, first, show that

$$
T_{i m}(X) \subset T_{i m}(\mathcal{Z}) .
$$

Suppose the contrary: there exists $\bar{t} \in T_{i m}(X)$ such that $\bar{t} \notin T_{i m}(\mathcal{Z})$. The latter relation implies the following condition:

$$
\exists\left(\tilde{y}, \tilde{y}_{0}\right) \in \mathcal{Z}: \bar{t}^{\top} \mathcal{B}\left(\tilde{y}, \tilde{y}_{0}\right) \bar{t}>0, \tilde{y}_{0} \geq 0 .
$$

In the case $\tilde{y}_{0}>0$, let us set $\bar{x}:=\tilde{y} / \tilde{y}_{0}$ and in the case $\tilde{y}_{0}=0$, set $\bar{x}:=x^{*}+\tilde{y}$, where $x^{*}$ is an arbitrary vector from $X$.

It is easy to check that by construction, we have $\bar{x} \in X$ and $\bar{t}^{\top} \mathcal{A}(\bar{x}) \bar{t}>0$, but this contradicts the assumption $\bar{t} \in T_{i m}(X)$. Hence, inclusion (17) is proved.

Now let us show that

$$
T_{i m}(\mathcal{Z}) \subset T_{i m}(X) .
$$

Suppose the contrary: there exists $\bar{t} \in T_{i m}(\mathcal{Z})$ such that $\bar{t} \notin T_{i m}(X)$. Then for some $\bar{x} \in X$, it holds that $\bar{t}^{\top} \mathcal{A}(\bar{x}) \bar{t}>0$.

Consider vector $\tilde{z}:=\left(\tilde{y}=\bar{x}, \tilde{y}_{0}=1\right)$. By construction, $\tilde{z} \in \mathcal{Z}$ and $\bar{t}^{\top} \mathcal{B}(\tilde{z}) \bar{t}>0$, but this contradicts the assumption $\bar{t} \in T_{i m}(\mathcal{Z})$. Hence, inclusion (18) is valid and the proposition is proved.

Proposition 3. Given $\tau(i) \in T_{\text {im }}(\mathcal{Z}), i \in I$, suppose that the set

$$
\Delta X:=\left\{x \in \mathbb{R}^{n}: \mathcal{A}(x) \tau(i) \geq 0 \forall i \in I\right\}
$$

is empty. Then the set $X$ is empty too.

Proof. Suppose the contrary: $\Delta X=\varnothing$ but $X \neq \varnothing$.

Since $X \neq \varnothing$, then by Proposition 2 we have $\tau(i) \in T_{i m}(X), i \in I$. Hence, according to Proposition 2 in [20], the following inequalities hold true: $\mathcal{A}(x) \tau(i) \geq 0 \forall i \in I$, for all $x \in X$. However, this contradicts the assumption that $\Delta X=\varnothing$. The proposition is proved. 
Proposition 4. Given a finite set $\{\tau(i), i \in I\} \subset T_{i m}(\mathcal{Z})$, suppose that there are numbers and vectors $\gamma_{i}>0, \tau(i) \in T, i \in \Delta I, \lambda(i) \in \mathbb{R}_{+}^{p}, i \in I$, such that

$$
\begin{aligned}
& \sum_{i \in \Delta I} \gamma_{i}(\tau(i))^{\top} A_{j} \tau(i)+\sum_{i \in I}(\lambda(i))^{\top} A_{j} \tau(i)=0 \forall j=1, \ldots, n ; \\
& \sum_{i \in \Delta I} \gamma_{i}(\tau(i))^{\top} A_{0} \tau(i)+\sum_{i \in I}(\lambda(i))^{\top} A_{0} \tau(i)=: \eta<0 .
\end{aligned}
$$

Then, $X=\varnothing$.

Proof. Suppose the contrary: relations (20) hold true and there exists $x^{*} \in X$. Then from Proposition 2 it follows that $\tau(i) \in T_{i m}(X) \forall i \in I$, and hence

$$
\mathcal{A}\left(x^{*}\right) \tau(i) \geq 0 \forall i \in I, t^{\top} \mathcal{A}\left(x^{*}\right) t \geq 0 \forall t \in T .
$$

Denote

$$
\beta_{*}:=\sum_{i \in \Delta I} \gamma_{i}(\tau(i))^{\top} \mathcal{A}\left(x^{*}\right) \tau(i)+\sum_{i \in I}(\lambda(i))^{\top} \mathcal{A}\left(x^{*}\right) \tau(i) .
$$

It follows from relations (21) that $\beta_{*} \geq 0$. On the other hand, from (20) we can conclude that $\beta_{*}=\eta<0$. The resulting contradiction implies that $X=\varnothing$. The proposition is proved.

Proposition 5. Given a set $W:=\{\tau(i), i \in I\} \subset T_{i m}(\mathcal{Z})$, the corresponding number $\sigma(W)$, and the sets $\Omega(W), \mathcal{X}(W)$ defined in (12) -(14), suppose that the set $\Delta X$ defined in (19) is not empty and for some vector $\left(\tilde{y}, \tilde{y}_{0}\right) \in \mathbb{R}^{n+1}$, it holds

$$
\tilde{y}_{0} \geq 0, \mathcal{B}\left(\tilde{y}, \tilde{y}_{0}\right) \tau(i) \geq 0, i \in I ; t^{\top} \mathcal{B}\left(\tilde{y}, \tilde{y}_{0}\right) t \geq \mu>0 \forall t \in \Omega(W)
$$

Then $\mathcal{X}(W)=X \neq \varnothing$ and there exists $\bar{x} \in X$ such that

$$
t^{\top} \mathcal{A}(\bar{x}) t>0 \forall t \in \Omega(W)
$$

Proof. First, let us show that $X \neq \varnothing$ and there exists $\bar{x} \in X$ such that inequalities (23) hold true. From (22) and Theorem 1, it follows that $\left(\tilde{y}, \tilde{y}_{0}\right) \in \mathcal{Z}$. Consider the following cases: (a) $\tilde{y}_{0}>0$ and (b) $\tilde{y}_{0}=0$.

In the case (a), set $\bar{x}:=\tilde{y} / \tilde{y}_{0}$. It is easy to see that, by construction, $\bar{x} \in X$ and inequalities (23) hold true.

Now consider the case (b). Here relations (22) take the form

$$
\left(\sum_{j=1}^{n} A_{j} \tilde{y}_{j}\right) \tau(i) \geq 0 \forall i \in I ; t^{\top}\left(\sum_{j=1}^{n} A_{j} \tilde{y}_{j}\right) t \geq \mu>0 \forall t \in \Omega(W) .
$$

For a fixed $x^{*} \in \Delta X$, set:

$$
\alpha_{*}:=\min _{t \in \Omega(W)} t^{\top} \mathcal{A}\left(x^{*}\right) t \text { and } \theta:=\left\{\begin{array}{cl}
1, & \text { if } \alpha_{*} \geq 0, \\
-2 \alpha_{*} / \mu>0, & \text { if } \alpha_{*}<0,
\end{array}\right.
$$

and consider vector $\bar{x}:=x^{*}+\theta \tilde{y}$. Taking into account relations (24) and the inclusion $x^{*} \in \Delta X$, we obtain

$$
\mathcal{A}(\bar{x}) \tau(i) \geq 0, i \in I, t^{\top} \mathcal{A}(\bar{x}) t \geq \alpha_{*}+\theta \mu=\left\{\begin{array}{cc}
\alpha_{*}+\mu>0, & \text { if } \alpha_{*} \geq 0, \\
-\alpha_{*}>0, & \text { if } \alpha_{*}<0,
\end{array} \quad \forall t \in \Omega(W)\right.
$$

From the relations above and from Theorem 1 in [18], it follows that $\bar{x} \in X$ and inequalities (23) hold true. 
Since $X \neq \varnothing$, from Proposition 2 we deduce that $T_{i m}(X)=T_{i m}(\mathcal{Z})$. This means that $W \subset T_{i m}(X)$, and hence according to Theorem 1 , we conclude that $X=\mathcal{X}(W)$. The proposition is proved.

\section{Regularization Algorithms Based on the Concept of Immobile Indices}

In this section, we present further developments of the approach proposed in [17], and construct and justify new explicit algorithms for regularization of linear copositive problems that are modifications of the algorithm REG-LCoP. These modifications are designated here as algorithms RLCoP-1, RLCoP-2, and RLCoP-3.

Notice that the modified algorithms have a structure similar to that of the algorithm REG-LCoP, but they do not require the feasibility of the original optimization problem due to the use of the properties of the copositive problem (1) established in this paper and based on the alternative representations of its feasible set. Although these representations are based on the properties of immobile indices, at the iterations of the algorithms we do not need to know in advance either the set of immobile indices or its convex hull.

\subsection{Regularization Algorithm RLCoP-1}

Based on the results of the previous section, we describe here in detail the iterations of the algorithm RLCoP-1.

Iteration \# 0. Given the copositive problem in the form (1), consider the following regular SIP problem:

$$
\operatorname{SIP}_{0}^{1}: \quad \max _{\left(y, y_{0}, \mu\right) \in \mathbb{R}^{n+2}} \mu \text {, s.t. } t^{\top} \mathcal{B}\left(y, y_{0}\right) t-\mu \geq 0 \forall t \in T, y_{0} \geq 0,
$$

with the compact index set $T$ defined in (4).

If there exists a feasible solution $\left(\bar{y}, \bar{y}_{0}, \bar{\mu}\right)$ of the problem $\left(S I P_{0}^{1}\right)$ with $\bar{\mu}>0$, then set $m_{*}:=0$ and go to the Final step.

Otherwise the vector $\left(y=\mathbf{0}, y_{0}=0, \mu=0\right)$ is an optimal solution of this problem.

By construction, in the problem $\left(S I P_{0}^{1}\right)$, the index set $T$ is compact, and the constraints satisfy the Slater condition. Hence, (see, e.g., [21]), it follows from the optimality conditions for the vector $\left(y=\mathbf{0}, y_{0}=0, \mu=0\right)$ that there exist indices and numbers

$$
\tau(i) \in T, \gamma(i)>0 \forall i \in I_{1}, \quad\left|I_{1}\right| \leq n+2,
$$

such that

$$
\begin{gathered}
\sum_{i \in I_{1}} \gamma(i)(\tau(i))^{\top} A_{j} \tau(i)=0 \forall j=1, \ldots, n ; \quad \sum_{i \in I_{1}} \gamma(i)=1, \\
\sum_{i \in I_{1}} \gamma(i)(\tau(i))^{\top} A_{0} \tau(i)=: \eta_{0} \leq 0 .
\end{gathered}
$$

If $\eta_{0}<0$, then STOP: from Proposition 4, it follows that $X=\varnothing$.

Suppose that $\eta_{0}=0$. From the relations (26) and (27) (with $\eta_{0}=0$ ), one can conclude that $I_{1} \neq \varnothing$ and $\tau(i) \in T_{i m}(\mathcal{Z}) \subset T$ for all $i \in I_{1}$. Go to the next iteration with the set of indices $W_{1}:=\left\{\tau(i), i \in I_{1}\right\}$.

Iteration $\# m, m \geq 1$. By the beginning of the iteration, we have the index set $W_{m}:=\left\{\tau(i), i \in I_{m}\right\}$, where

$$
\tau(i) \in T_{i m}(\mathcal{Z}) \forall i \in I_{m} .
$$

Consider a SIP problem

$$
\begin{array}{cc} 
& \max _{\left(y, y_{0}, \mu\right) \in \mathbb{R}^{n+2}} \mu, \\
\operatorname{SIP}_{m}^{1}: \quad \text { s.t. } t^{\top} \mathcal{B}\left(y, y_{0}\right) t-\mu \geq 0 \forall t \in \Omega\left(W_{m}\right) ; \\
\mathcal{B}\left(y, y_{0}\right) \tau(i) \geq 0 \forall i \in I_{m}, y_{0} \geq 0,
\end{array}
$$


where the set $\Omega\left(W_{m}\right)$ is constructed by the rules (12) and (13) with $W=W_{m}$.

Since $W_{m}$ is a subset of the set of immobile indices of $\mathcal{Z}$, according to Theorem 1 , we conclude that $\mathcal{Z}\left(W_{m}\right)=\mathcal{Z}$, where the set $\mathcal{Z}(W)$ is defined in (15).

Notice that by the definition of the set $\Omega\left(W_{m}\right)$, it holds

$$
\rho\left(t, \operatorname{conv} W_{m}\right) \geq \sigma\left(W_{m}\right)>0 \quad \forall t \in \Omega\left(W_{m}\right) .
$$

If the problem $\left(S I P_{m}^{1}\right)$ admits a feasible solution $\left(\bar{y}, \bar{y}_{0}, \bar{\mu}\right)$ with $\bar{\mu}>0$, then go to the Final step with $m_{*}:=m$ or else, the vector $\left(y=\mathbf{0}, y_{0}=0, \mu=0\right)$ is an optimal solution of this problem.

In the problem $\left(S I P_{m}^{1}\right)$, the constraints $\mathcal{B}\left(y, y_{0}\right) \tau(i) \geq 0 \forall i \in I_{m}$, are linear, the index set $\Omega\left(W_{m}\right)$ is compact, and the following Slater type condition is satisfied:

$$
\begin{aligned}
& \exists\left(\widehat{y}, \widehat{y}_{0}, \widehat{\mu}\right) \text { such that } \mathcal{B}\left(\widehat{y}, \widehat{y}_{0}\right) \tau(i) \geq 0 \forall i \in I_{m} ; \\
& \qquad t^{\top} \mathcal{B}\left(\widehat{y}, \widehat{y}_{0}\right) t-\widehat{\mu}>0 \forall t \in \Omega\left(W_{m}\right), \widehat{y}_{0} \geq 0 .
\end{aligned}
$$

Due to these properties, the optimality of the vector mentioned above provides (see [22]) that there exist indices, numbers, and vectors

$$
\tau(i) \in \Omega\left(W_{m}\right), \gamma(i)>0, i \in \Delta I_{m}, 1 \leq\left|\Delta I_{m}\right| \leq n+2 ; \lambda^{m}(i) \in \mathbb{R}_{+}^{p}, i \in I_{m},
$$

satisfying the following conditions:

$$
\begin{gathered}
\sum_{i \in \Delta I_{m}} \gamma(i)(\tau(i))^{\top} A_{j} \tau(i)+\sum_{i \in I_{m}}\left(\lambda^{m}(i)\right)^{\top} A_{j} \tau(i)=0 \forall j=1, \ldots, n ; \\
\sum_{i \in \Delta I_{m}} \gamma(i)(\tau(i))^{\top} A_{0} \tau(i)+\sum_{i \in I_{m}}\left(\lambda^{m}(i)\right)^{\top} A_{0} \tau(i)=: \eta_{m} \leq 0 .
\end{gathered}
$$

Moreover, by applying the procedure DAM described in [20], to the data presented in (28), it is possible to ensure that the following conditions are met:

$$
\left(P \backslash P_{+}(\tau(i))\right) \cap P_{+}(\tau(j)) \neq \varnothing \forall i \in \Delta I_{m}, \forall j \in I_{m} .
$$

If $\eta_{m}<0$, then STOP: it follows from Proposition 4 that $X=\varnothing$.

Suppose that $\eta_{m}=0$. Then from (29) and (30) we get $\tau(i) \in T_{i m}(\mathcal{Z}) \forall i \in \Delta I_{m}$. Go to the next iteration $\#(m+1)$ with the new index set

$$
W_{m+1}:=\left\{\tau(i), i \in I_{m+1}:=I_{m} \cup \Delta I_{m}\right\} .
$$

It follows from (31) that the RLCoP- 1 algorithm executes a finite number $m_{*}$ of iterations, during which it either recognizes that $X=\varnothing$ or proceeds to the final step.

Final step. At this step, we have that for some $m_{*} \geq 0$, the problem $\left(S I P_{m_{*}}^{1}\right)$ has a feasible solution $\left(\bar{y}, \bar{y}_{0}, \bar{\mu}\right)$ with $\bar{\mu}>0$. Observe that by Theorem 1 , it holds $\left(\bar{y}, \bar{y}_{0}\right) \in \mathcal{Z}$.

If $m_{*}=0$, then the set $T_{i m}(\mathcal{Z})$ is empty. From Proposition 1 , we conclude that $X \neq \varnothing$ and $T_{i m}(X)=\varnothing$. Hence, problem (1) is regular (its constraints satisfy the Slater condition).

Suppose now that $m_{*}>0$. By construction, the following inequalities hold true for the found vector $\left(\bar{y}, \bar{y}_{0}\right) \in \mathcal{Z}$ :

$$
\bar{y}_{0} \geq 0, \mathcal{B}\left(\bar{y}, \bar{y}_{0}\right) \tau(i) \geq 0, i \in I_{m_{*}} ; t^{\top} \mathcal{B}\left(\bar{y}, \bar{y}_{0}\right) t \geq \mu>0 \forall t \in \Omega\left(W_{m_{*}}\right),
$$

where the sets $W_{m_{*}}=\left\{\tau(i), i \in I_{m_{*}}\right\}$ and $\Omega\left(W_{m_{*}}\right)$ are the same as in the problem $\left(\operatorname{SIP}_{m_{*}}^{1}\right)$.

Consider the set

$$
\Delta X_{*}:=\left\{x \in \mathbb{R}^{n}: \mathcal{A}(x) \tau(i) \geq 0 \forall i \in I_{m_{*}}\right\} .
$$

If $\Delta X_{*}=\varnothing$, then from Proposition 3 it follows that $X=\varnothing$. 
Suppose that $\Delta X_{*} \neq \varnothing$. Taking into account (33) and Proposition 5, we conclude that $\mathcal{X}\left(W_{m_{*}}\right)=X \neq \varnothing$ (recall that the set $\mathcal{X}(W)$ is defined in (14)) and

$$
\exists \bar{x} \in X \text { such that } t^{\top} \mathcal{A}(\bar{x}) t>0 \forall t \in \Omega\left(W_{m_{*}}\right) .
$$

Consider a SIP problem

$$
R P: \quad \min _{x \in \mathbb{R}^{n}} c^{\top} x \text {, s.t. } t^{\top} \mathcal{A}(x) t \geq 0 \forall t \in \Omega\left(W_{m_{*}}\right), \mathcal{A}(x) \tau(i) \geq 0 \forall i \in I_{m_{*}} .
$$

This problem is equivalent to the copositive problem (1) and can be considered as its regularization since, by construction,

- $\mathcal{X}\left(W_{m_{*}}\right)=X$, and hence the problems (1) and $(R P)$ have the same feasible sets;

- relations (35) hold true, and hence the first group of constraints in $(R P)$ satisfies the Slater condition;

- the inequalities in the second group of constraints are formulated in terms of linear functions and the number of these constraints is finite.

Therefore, the regularized problem is constructed and the algorithm is described.

Thanks to the above-mentioned properties of the problem $(R P)$, it is easy to formulate for this problem the KKT optimality conditions and the Lagrange dual problem satisfying the strong duality relations. It is widely known (see, e.g., [23-25]) that all of these properties are quite useful for creating numerical methods (such as methods based on discretization and interior point methods).

To give a different interpretation of the RLCoP- 1 algorithm and facilitate the comparison of this method with other FRAs and the modifications of the REG-LCoP algorithm described in what follows, let us display some additional constructions for its iterations.

Set $\mathcal{F}_{0}^{1}:=\mathcal{C O} \mathcal{P}^{p}$.

Suppose that $m_{*} \geq 1$. For all $m: 1 \leq m \leq m_{*}$, at the beginning of Iteration \# $m$, we have the index set $W_{m}=\left\{\tau(i), i \in I_{m}\right\}$. Let us denote

$$
\mathcal{F}_{m}^{1}:=\left\{D \in \mathcal{C O P}^{p}: D \tau(i) \geq 0 \forall i \in I_{m}\right\} .
$$

By construction, all the sets $\mathcal{F}_{m}^{1}$, are convex cones such that $\mathcal{D} \subset \mathcal{F}_{m}^{1} \subset \mathcal{F}_{m-1}^{1}$ for $m=1, \ldots m_{*}$, where the set $\mathcal{D}$ is defined in (6). Note that in general, for $m=0,1, \ldots, m_{*}$, the cone $\mathcal{F}_{m}^{1} \subset \mathcal{C O P}{ }^{p}$ is not a face of $\mathcal{C O P} \mathcal{P}^{p}$.

\subsection{Regularization Algorithm RLCoP-2}

To get another one modification of the algorithm REG-LCoP, let us introduce the following changes to the RLCoP-1 algorithm presented above.

For $m \geq 1$, replace the auxiliary problem $\left(S I P_{m}^{1}\right)$ with the following one:

$$
\begin{aligned}
& \max _{\left(y, y_{0}, \mu\right) \in \mathbb{R}^{n+2}} \mu ， \\
& S I P_{m}^{2}: \quad \text { s.t. } t^{\top} \mathcal{B}\left(y, y_{0}\right) t-\mu \geq 0 \forall t \in \Omega\left(W_{m}\right) ; y_{0} \geq 0 \text {, } \\
& e_{k}^{\top} \mathcal{B}\left(y, y_{0}\right) \tau(i)=0, k \in P_{+}(\tau(i)), \\
& e_{k}^{\top} \mathcal{B}\left(y, y_{0}\right) \tau(i) \geq 0, k \in P \backslash P_{+}(\tau(i)), \forall i \in I_{m},
\end{aligned}
$$

where $\left\{e_{k}, k \in P\right\}$ is the canonic basis of $\mathbb{R}^{p}$.

If for this problem there is a feasible solution $\left(\bar{y}, \bar{y}_{0}, \bar{\mu}\right)$ with $\bar{\mu}>0$, then proceed to the final step which coincides with the final step of the algorithm RLCoP-1; if not, the vector $\left(y=\mathbf{0}, y_{0}=0, \mu=0\right)$ is an optimal solution of $\left(S I P_{m}^{2}\right)$ and therefore there are indices, numbers, and vectors.

$$
\begin{gathered}
\tau(i) \in \Omega\left(W_{m}\right), \gamma(i)>0, i \in \Delta I_{m}, \quad 1 \leq\left|\Delta I_{m}\right| \leq n+1 ; \\
\lambda^{m}(i) \in \mathbb{R}^{p}, \lambda_{k}^{m}(i) \geq 0 \forall k \in P \backslash P_{+}(\tau(i)), i \in I_{m},
\end{gathered}
$$


satisfying conditions (29) and (30).

If $\eta_{m}<0$ (see (30)), then STOP: $X=\varnothing$. Otherwise, go to the next Iteration \# $(m+1)$ with the new index set $W_{m+1}$ defined in (32). The algorithm is described.

As before, for comparison, we will present additional constructions.

Set $\mathcal{F}_{0}^{2}:=\mathcal{C O} \mathcal{P}^{p}$. Suppose that $m_{*} \geq 1$. Then for any $m: 1 \leq m \leq m_{*}$, using the index set $W_{m}=\left\{\tau(i), i \in I_{m}\right\}$ available at the beginning of Iteration $\#$, we may construct a set

$$
\mathcal{F}_{m}^{2}:=\left\{D \in \mathcal{C O} \mathcal{P}^{p}: e_{k}^{\top} D \tau(i)=0 \forall k \in P_{+}(\tau(i)), \forall i \in I_{m}\right\} .
$$

Notice here that by construction, all the sets $\mathcal{F}_{m}^{2}, m=0,1, \ldots, m_{*}$, are exposed faces of $\mathcal{C O P}^{p}$ (see Remark 1 in [18]) and $\mathcal{D} \subset \mathcal{F}_{m}^{2} \subset \mathcal{F}_{m-1}^{2}$ for all $m=1,2, \ldots, m_{*}$.

\subsection{Regularization Algorithm RLCoP-3}

Iteration \# 0 of this modification of the REG-LCoP algorithm coincides with Iteration \# 0 of the algorithm RLCoP-1.

For $m \geq 1$, let $W_{m}=\left\{\tau(i), i \in I_{m}\right\}$ be the index set found at the end of Iteration $\#(m-1)$ and available at the beginning of Iteration \#m. Denote

$$
\begin{aligned}
& Z(m):=\left\{\left(y, y_{0}\right) \in \mathbb{R}^{n+1}: \mathcal{B}\left(y, y_{0}\right) \tau(i) \geq 0 \forall i \in I_{m} ; y_{0} \geq 0\right\}, \\
& L^{m}(i):=\left\{k \in P: e_{k}^{\top} \mathcal{B}\left(y, y_{0}\right) \tau(i)=0 \forall\left(y, y_{0}\right) \in Z(m)\right\}, \\
& S^{m}(i):=P \backslash L^{m}(i), i \in I_{m} .
\end{aligned}
$$

Note that the set $Z(m)$ is polyhedral. Hence, given $i \in I_{m}$, to construct the set $L^{m}(i)$ it is enough to solve $p_{0}$ (with $p_{0}<p$ ) linear programming problems.

Consider the following semi-infinite problem:

$$
\begin{gathered}
\max _{\left(y, y_{0}, \mu\right) \in \mathbb{R}^{n+2}} \mu, \\
\text { SIP } P_{m}^{3}: \quad \text { s.t. } t^{\top} \mathcal{B}\left(y, y_{0}\right) t-\mu \geq 0 \forall t \in \Omega\left(W_{m}\right), y_{0} \geq 0 ; \\
e_{k}^{\top} \mathcal{B}\left(y, y_{0}\right) \tau(i)=0, k \in L^{m}(i), \\
\\
e_{k}^{\top} \mathcal{B}\left(y_{1}, y_{0}\right) \tau(i) \geq 0, k \in S^{m}(i), \forall i \in I_{m} .
\end{gathered}
$$

If for this problem there is a feasible solution $\left(\bar{y}, \bar{y}_{0}, \bar{\mu}\right)$ with $\bar{\mu}>0$, then set $m_{*}:=m$ and go to the final step, which is the same as that of the RLCoP-1 algorithm.

Otherwise, the vector $\left(y=\mathbf{0}, y_{0}=0, \mu=0\right)$ is an optimal solution of the problem $\left(S I P_{m}^{3}\right)$ and therefore there are indices, numbers, and vectors

$$
\begin{gathered}
\tau(i) \in \Omega\left(W_{m}\right), \gamma(i)>0, i \in \Delta I_{m}, \quad 1 \leq\left|\Delta I_{m}\right| \leq n+2 ; \\
\lambda^{m}(i) \in \mathbb{R}^{p}, \lambda_{k}^{m}(i) \geq 0 \forall k \in S^{m}(i), i \in I_{m},
\end{gathered}
$$

satisfying conditions (29) and (30).

If $\eta_{m}<0$, then STOP: $X=\varnothing$. Otherwise, go to the next Iteration $\#(m+1)$ with the new index set $W_{m+1}$ defined in (32). The algorithm is described.

Set $\mathcal{F}_{0}^{3}:=\mathcal{C O} \mathcal{P}^{p}$. Suppose that $m_{*} \geq 1$. For $m: 1 \leq m \leq m_{*}$, using the index set $W_{m}=\left\{\tau(i), i \in I_{m}\right\}$, available at the beginning of Iteration $\# m$, and the sets $L^{m}(i), i \in I_{m}$, defined in (37). Then we may construct the set

$$
\mathcal{F}_{m}^{3}:=\left\{D \in \mathcal{C O P}^{p}: e_{k}^{\top} D \tau(i)=0 \forall k \in L^{m}(i), \forall i \in I_{m}\right\} .
$$

In the Appendix A.2, it is shown that $P_{+}(\tau(i)) \subset L^{m}(i), i \in I_{m}$, and hence (see Proposition 2 in [18]) all the sets $\mathcal{F}_{m}^{3}, m=0,1, \ldots, m_{*}$, are faces of $\mathcal{C O} \mathcal{P}^{p}$.

By construction, $\mathcal{D} \subset \mathcal{F}_{m}^{3} \subset \mathcal{F}_{m-1}^{3}$ for all $m=1,2, \ldots, m_{*}$. 
Remark 1. For all modifications of the algorithm REG-LCoP described above in this section, the number of iterations required to either recognize the fact that $X=\varnothing$ or obtain a regularized problem may be different, but to simplify the presentation, we will denote everywhere the number of iterations by $m_{*}$.

The following proposition and lemma characterizing properties of the proposed algorithms are proved in the Appendices A.1 and A.3.

Proposition 6. Suppose that $X \neq \varnothing$. Let $W_{m_{*}}=\left\{\tau(i), i \in I_{m_{*}}\right\}$ be the set of indices constructed at the final iteration of the algorithm $R L C o P-k$, where $k \in\{1,2,3\}$. Then the following inclusion is satisfied:

$$
\left\{\tau^{*}(j), j \in J\right\} \subset W_{m_{*}}
$$

where $\left\{\tau^{*}(j), j \in J\right\}$ is the set of all vertices of the set conv $T_{i m}(X)$.

Lemma 2. Suppose that $X \neq \varnothing$, and let $m_{*}$ be the number of the final iteration of the algorithm $R L C o P-3$. Then $\mathcal{F}_{m_{*}}^{3}=\mathbf{F}_{\text {min }}$, where $\mathcal{F}_{m_{*}}^{3}$ is the set defined by the rules (39) and $\mathbf{F}_{\text {min }}$ is the minimal face containing the set $\mathcal{D}$ given in (6).

Note that in [17], it was shown that in iterations of the algorithm REG-LCoP, some sets, $F_{m}, m=0,1, \ldots, m_{*}$, are constructed. These sets are subcones of $\mathcal{C O} \mathcal{P}^{p}$ and it holds that $\mathcal{D} \subset F_{m} \subset F_{m-1}$ for $m=1, \ldots, m_{*}$.

The main differences between the algorithm RLCoP-k, $k=1,2,3$, described in this paper, and the algorithm REG-LCoP from [17], are the auxiliary problems that are solved in their iterations and the properties of the sets $\mathcal{F}_{m}^{k}, k=1,2,3, F_{m}, m=0,1, \ldots, m_{*}$, constructed on the basis of solutions to these problems.

Suppose that $X \neq \varnothing$, and let $\mathcal{F}_{m_{*}}^{k}, k=1,2,3, F_{m_{*}}$ be the sets constructed in the final iterations of algorithms RLCoP-k, $k=1,2,3$, and REG-LCoP. It was shown that $\mathcal{F}_{m_{*}}^{3}$ is the minimal face containing the set $\mathcal{D}$ defined in (6), the set $\mathcal{F}_{m_{*}}^{2}$ is an exposed face (in general, it is not the minimal exposed face containing the set $\mathcal{D}$ ), the set $\mathcal{F}_{m_{*}}^{1} \subset \mathcal{C O} \mathcal{P}^{p}$ is a cone (in general, it is not a face of $\mathcal{C O} \mathcal{P}^{p}$ ), and the set $F_{m_{*}} \subset \mathcal{C O} \mathcal{P}^{p}$ is a face (in general it is not a minimal face containing $\mathcal{D}$ ). It is easy to prove that

$$
\mathcal{D} \subset \mathcal{F}_{m_{*}}^{3} \subset F_{m_{*}} \subset \mathcal{F}_{m_{*}}^{2} \subset \mathcal{F}_{m_{*}}^{1}
$$

To finalize, let us compare the modifications of the algorithm REG-LCoP proposed in this section with the FRA algorithm from [12], which is the most suitable comparison for our method that can be found in the literature.

Given the copositive problem (1), the FRA algorithm from [12] generates a set of smaller faces $\mathbf{F}_{m}, m=0,1, \ldots, m_{*}$ of $\mathcal{C O} \mathcal{P}^{p}$ until it stops with the minimal face $\mathbf{F}_{\text {min }}$.

Being reformulated for problem (1), the whole algorithm from [12] takes only few lines and consists of the following steps.

Step 1: Set $m:=0$ and $\mathbf{F}_{0}:=\mathcal{C O} \mathcal{P}^{p}$.

Step 2: If $\operatorname{Ker} \mathcal{A} \cap H_{c}^{-} \cap \mathbf{F}_{m}^{*} \subset \operatorname{span}\left\{Y_{1}, \ldots, Y_{m}\right\}$, then STOP: $\mathbf{F}_{\text {min }}=\mathbf{F}_{m}$.

Step 3: Find $Y_{m+1} \in \operatorname{Ker} \mathcal{A} \cap H_{c}^{-} \cap \mathbf{F}_{m}^{*} \backslash \operatorname{span}\left\{Y_{1}, \ldots, Y_{m}\right\}$.

Step 4: If trace $\left(A_{0} Y_{m+1}\right)<0$, then STOP. The problem (1) is infeasible.

Step 5: Set $\mathbf{F}_{m+1}:=\mathbf{F}_{m} \cap\left\{Y_{i+1}\right\}^{\perp}, m:=m+1$, and go to step 2 .

Here $\mathbf{F}_{m}^{*}$ is the cone dual to $\mathbf{F}_{m}, \operatorname{Ker} \mathcal{A}:=\left\{Y \in \mathcal{S}^{p}: \operatorname{trace}\left(A_{j} Y\right)=0 \forall j=1, \ldots, n\right\}$, $H_{c}^{-}=\left\{Y \in \mathcal{S}^{p}: \operatorname{trace}\left(A_{0} Y\right) \leq 0\right\}$.

It should be noted that the description of this algorithm looks rather simple, but in practice, its implementation presents serious difficulties, since there is no explicit description of the dual cone $\mathbf{F}_{m}^{*}$ and there is no information concerning how to find a matrix $Y_{m+1}$ at the step 3, where $m$ is the number of the current iteration. 
Unlike the FRA algorithm from [12], using the output data of Iteration \# $m$, of each algorithm RLCoP-k, $k=1,2,3$, described here, the subcone $\mathcal{F}_{m}^{k}$ of the cone $\mathcal{C} \mathcal{O} \mathcal{P}^{p}$ can be constructed on the basis of the optimality conditions for the feasible solution $(x=\mathbf{0}, \mu=0)$ in the corresponding regular semi-infinite problem $\left(S I P_{m}^{k}\right)$.

For a more detailed comparison of the basic algorithm REG-LCoP and the FRA algorithm ([12]), the interested reader can see the discussion in [17].

The purpose of this work was to develop a theoretical and constructive basis for further development of theory and explicit regularization algorithms for CoP. The results of the paper and the form in which the modified algorithms were constructed permit us to stress the algorithmic aspects of our approach. On other hand, we still need to develop several new computational procedures that allow practical implementations and numerical testing of our methods. In particular, at iterations of the algorithms RLCoP-k, $k=1,2,3$, we solve the corresponding regular SIP problems $\left(S I P_{m}^{k}\right)$. Although there already exist some SIP solvers, for effective numerical implementation of our algorithms, we will need special solvers taking into account the specific structures of the corresponding SIP problems.

\section{Conclusions}

The main contributions of the paper were further developments of the regularization approach for linear CoP. This approach is based on the concept of immobile indices previously introduced for semi-infinite and semidefinite optimization problems. It is important to stress that, at the moment, no constructive regularization procedures are known for linear copositive problems. The modifications we presented of the regularization algorithm REG-LCoP from [17] are new and take into account the specific features of CoP problem. An important property of these modified algorithms is that they require neither the feasibility of the problem nor additional information about the immobile index set. In future, these algorithms could be implemented in the form of computational algorithms and also used for further study of CoP problems. In particular, for linear copositive problems, the results of this paper allow one to formulate and prove CQ-free optimality conditions and develop a strong duality theory based on the explicit representation of the "regularized" feasible cones.

Author Contributions: Conceptualization, O.K. and T.T.; methodology, O.K.; formal analysis, O.K. and T.T.; investigation, O.K. and T.T.; writing-original draft preparation, O.K. and T.T.; writingreview and editing, O.K. and T.T. All authors have read and agreed to the published version of the manuscript.

Funding: This research was partially supported by the state research program "Convergence" (Republic Belarus), Task 1.3.01, and by Portuguese funds through CIDMA-Center for Research and Development in Mathematics and Applications, and FCT-Portuguese Foundation for Science and Technology, within the project UIDB/04106/2020.

Institutional Review Board Statement: Not applicable.

Informed Consent Statement: Not applicable.

Data Availability Statement: Not applicable.

Conflicts of Interest: The authors declare no conflict of interest.

\section{Abbreviations}

The following abbreviations are used in this manuscript:

$\begin{array}{ll}\text { CoP } & \text { Copositive Programming } \\ \text { SIP } & \text { Semi-Infimite Programming } \\ \text { SDP } & \text { Semidefinite Programming } \\ \text { FRA } & \text { Facial Reduction Algorithm } \\ \text { CQ } & \text { Constraint Qualification } \\ \text { KKT-type conditions } & \text { Karush-Kuhn-Tucker-Type Conditions }\end{array}$




\section{Appendix A}

Appendix A.1.

In this subsection we give a proof of Proposition 6.

First of all, notice that since $X \neq \varnothing$, the set $\Delta X_{*}$ defined in (34) is not empty and from Proposition 5 it follows that $T_{i m}(X)=T_{i m}(\mathcal{Z})$ and relations (35) hold true.

Suppose that on the contrary to (40), there exists $j_{0} \in J$ such that $\tau^{*}\left(j_{0}\right) \notin W_{m_{*}}$. Since $\tau^{*}\left(j_{0}\right) \in T_{i m}(X)$ and by construction relations (35) hold true, we conclude that

$$
\rho\left(\tau^{*}\left(j_{0}\right), \operatorname{conv} W_{m_{*}}\right)<\sigma\left(W_{m_{*}}\right) .
$$

Hence, from Proposition 1 in [18], it follows that there exists $i_{0} \in I_{m_{*}}$ such that

$$
P_{+}\left(\tau^{*}\left(j_{0}\right)\right) \subset P_{+}\left(\tau\left(i_{0}\right)\right) \text { and } \tau^{*}\left(j_{0}\right) \neq \tau\left(i_{0}\right) .
$$

Then for all $x \in X$, it holds that

$$
\left(\tau^{*}\left(j_{0}\right)\right)^{\top} \mathcal{A}(x) \tau\left(i_{0}\right)=\sum_{k \in P_{+}\left(\tau^{*}\left(j_{0}\right)\right)} \tau_{k}^{*}\left(j_{0}\right) e_{k}^{\top} \mathcal{A}(x) \tau\left(i_{0}\right)=0,
$$

where $\tau_{k}^{*}\left(j_{0}\right)$ is the $k$-th component of the vector $\tau^{*}\left(j_{0}\right)$.

For a sufficiently small $\theta>0$, consider a vector $\bar{\tau}:=\left(\tau\left(i_{0}\right)-\theta \tau^{*}\left(j_{0}\right)\right) /(1-\theta)$. It is evident that $\bar{\tau} \in T$. Taking into account (A1), we conclude that for all $x \in X$, it holds that

$$
\begin{aligned}
\bar{\tau}^{\top} \mathcal{A}(x) \bar{\tau}= & (1-\theta)^{-2}\left[\left(\tau\left(i_{0}\right)\right)^{\top} \mathcal{A}(x) \tau\left(i_{0}\right)\right. \\
& \left.-2 \theta\left(\tau^{*}\left(j_{0}\right)\right)^{\top} \mathcal{A}(x) \tau\left(i_{0}\right)+\theta^{2}\left(\tau^{*}\left(j_{0}\right)\right)^{\top} \mathcal{A}(x) \tau^{*}\left(j_{0}\right)\right]=0,
\end{aligned}
$$

and therefore, $\bar{\tau} \in T_{i m}(X)$. As a result, we have

$$
\tau^{*}\left(j_{0}\right)=(1-\theta) \bar{\tau}+\theta \tau\left(i_{0}\right) \text { with } \bar{\tau} \in T_{i m}(X), \tau\left(i_{0}\right) \in T_{i m}(X), \bar{\tau} \neq \tau_{i_{0}}^{*} \text { and } \theta \in(0,1),
$$

which contradicts the assumption that $\tau^{*}\left(j_{0}\right)$ is a vertex of the set conv $T_{i m}(X)$. The proposition is proved.

Appendix A.2.

Consider the algorithm RLCoP-3 and suppose that $m_{*}>0$. Let us show that for all $m=1, \ldots, m_{*}$, it holds that

$$
P_{+}(\tau(i)) \subset L^{m}(i) \forall i \in I_{m} .
$$

At the end of Iteration \#0 we have data (25) satisfying relations (26) and (27) with $\eta_{0}=0$. It follows from these relations that

$$
\sum_{i \in I_{1}} \gamma(i)(\tau(i))^{\top} \mathcal{B}\left(y, y_{0}\right) \tau(i)=0 \forall\left(y, y_{0}\right) \in \mathbb{R}^{n+1} .
$$

Consider the set $Z(1):=\left\{\left(y, y_{0}\right) \in \mathbb{R}^{n+1}: \mathcal{B}\left(y, y_{0}\right) \tau(i) \geq 0, i \in I_{1}\right\}$. It is evident that by construction,

$$
(\tau(i))^{\top} \mathcal{B}\left(y, y_{0}\right) \tau(i) \geq 0 \forall i \in I_{1}, \forall\left(y, y_{0}\right) \in Z(1) .
$$

From the inequalities above and equality (A3), it follows that:

$$
(\tau(i))^{\top} \mathcal{B}\left(y, y_{0}\right) \tau(i)=0 \forall i \in I_{1}, \forall\left(y, y_{0}\right) \in Z(1),
$$

wherefrom we get

$$
e_{k}^{\top} \mathcal{B}\left(y, y_{0}\right) \tau(i)=0 \forall k \in P_{+}(\tau(i)), \forall i \in I_{1}, \forall\left(y, y_{0}\right) \in Z(1) .
$$


This implies that $P_{+}(\tau(i)) \subset L^{1}(i) \forall i \in I_{1}$.

Suppose that at the beginning of Iteration $\# m, 1 \leq m<m_{*}$, we have the sets $Z(m)$, $S^{m}(i), L^{m}(i), i \in I_{m}$, constructed by the rules (36) and (37), such that

$$
e_{k}^{\top} \mathcal{B}\left(y, y_{0}\right) \tau(i)=0 \forall k \in L^{m}(i), \forall\left(y, y_{0}\right) \in Z(m), P_{+}(\tau(i)) \subset L^{m}(i), \forall i \in I_{m} .
$$

At the end of this iteration, we have the data presented in (38) which satisfy relations (29) and (30) with $\eta_{m}=0$. These relations imply the equalities

$$
\sum_{i \in \Delta I_{m}} \gamma(i)(\tau(i))^{\top} \mathcal{B}\left(y, y_{0}\right) \tau(i)+2 \sum_{i \in I_{m}}\left(\lambda^{m}(i)\right)^{\top} \mathcal{B}\left(y, y_{0}\right) \tau(i)=0 \forall\left(y, y_{0}\right) \in \mathbb{R}^{n+1} \text {. }
$$

Set $I_{m+1}:=I_{m} \cup \Delta I_{m}$, and consider the set

$$
Z(m+1):=\left\{\left(y, y_{0}\right) \in \mathbb{R}^{n+1}: \mathcal{B}\left(y, y_{0}\right) \tau(i) \geq 0 \forall i \in I_{m+1}, y_{0} \geq 0\right\}
$$

Notice that by construction, $Z(m+1) \subset Z(m)$. The latest inclusion and (A4) imply

$$
\begin{aligned}
& e_{k}^{\top} \mathcal{B}\left(y, y_{0}\right) \tau(i)=0 \forall k \in L^{m}(i), \\
& e_{k}^{\top} \mathcal{B}\left(y, y_{0}\right) \tau(i) \geq 0 \forall k \in S^{m}(i), \forall i \in I_{m}, \forall\left(y, y_{0}\right) \in Z(m+1), \\
& (\tau(i))^{\top} \mathcal{B}\left(y, y_{0}\right) \tau(i) \geq 0 \forall i \in \Delta I_{m}, \forall\left(y, y_{0}\right) \in Z(m+1) .
\end{aligned}
$$

From the latter relations, conditions (38), and equalities (A5), it follows that

$$
(\tau(i))^{\top} \mathcal{B}\left(y, y_{0}\right) \tau(i)=0 \forall i \in \Delta I_{m}, \forall\left(y, y_{0}\right) \in Z(m+1)
$$

wherefrom we get

$$
e_{k}^{\top} \mathcal{B}\left(y, y_{0}\right) \tau(i)=0 \forall k \in P_{+}(\tau(i)), \forall i \in \Delta I_{m}, \forall\left(y, y_{0}\right) \in Z(m+1) \text {. }
$$

From the relation $Z(m+1) \subset Z(m)$, it follows that $L^{m}(i) \subset L^{m+1}(i) \forall i \in I_{m}$. Taking into account these inclusions, relations (A4) and (A6), we conclude that $P_{+}(\tau(i)) \subset L^{m+1}(i)$ $\forall i \in I_{m+1}$. Relations (A2) are proved.

\section{Appendix A.3.}

The aim of this subsection is to prove Lemma 2. The proof is based on Proposition 6 and the following one.

Proposition A1. Suppose that $X \neq \varnothing$. Let $W_{*}:=\left\{\tau^{*}(j), j \in J\right\}$ be the set of all vertices of the set conv $T_{i m}(X)$. Consider the sets

$$
\begin{gathered}
Z_{*}:=\left\{x \in \mathbb{R}^{n}: \mathcal{A}(x) \tau^{*}(j) \geq 0, j \in J\right\}, \\
\bar{M}(j):=\left\{k \in P: e_{k}^{\top} \mathcal{A}(x) \tau^{*}(j)=0 \forall x \in Z_{*}\right\}, j \in J .
\end{gathered}
$$

Then the set

$$
F_{*}:=\left\{D \in \mathcal{C O} \mathcal{P}^{p}: e_{k}^{\top} D \tau^{*}(j)=0, k \in \bar{M}(j), j \in J\right\}
$$

is the minimal face containing the set $\mathcal{D}$ defined in (6).

Proof. In our previous works, it has been shown (see Theorem 4 in [18]) that the minimal face $\mathbf{F}_{\min }$ containing the set $\mathcal{D}$ can be presented as follows:

$$
\mathbf{F}_{\min }=\left\{D \in \mathcal{C O} \mathcal{P}^{p}: e_{k}^{\top} D \tau^{*}(j)=0, k \in M(j), j \in J\right\},
$$

where $M(j)=\left\{k \in P: e_{k}^{\top} \mathcal{A}(x) \tau^{*}(j)=0 \forall x \in X\right\}, j \in J$. 
Since $X \subset Z_{*}, \bar{M}(j) \subset M(j)$ for all $j \in J$. Let us prove that

$$
\bar{M}(j)=M(j) \forall j \in J .
$$

Suppose the contrary: there exist $j_{0} \in J$ and $k_{0} \in M\left(j_{0}\right)$ such that $k_{0} \notin \bar{M}\left(j_{0}\right)$. In this case, there exists $\tilde{x} \in Z_{*}$ such that

$$
\mathcal{A}(\tilde{x}) \tau^{*}(j) \geq 0 \forall j \in J, e_{k_{0}}^{\top} \mathcal{A}(\tilde{x}) \tau^{*}\left(j_{0}\right)>0 .
$$

It was shown in [18] (see Theorem 2 there) that there exists $\bar{x} \in X$ such that

$$
t^{\top} \mathcal{A}(\bar{x}) t>0 \forall t \in \Omega\left(W_{*}\right), \mathcal{A}(\bar{x}) \tau^{*}(j) \geq 0 \forall j \in J .
$$

For a sufficiently small $\theta>0$, let us consider a vector $x(\theta):=(1-\theta) \bar{x}+\theta \tilde{x}$. From (A10) and (A11) we get

$$
\begin{gathered}
t^{\top} \mathcal{A}(x(\theta)) t \geq 0 \forall t \in \Omega\left(W_{*}\right), \mathcal{A}(x(\theta)) \tau^{*}(j) \geq 0 \forall j \in J, \\
e_{k_{0}}^{\top} \mathcal{A}(x(\theta)) \tau^{*}\left(j_{0}\right)>0 .
\end{gathered}
$$

From (A12) and Theorem 1 in [18], it follows that $x(\theta) \in X$. Then from (A13), taking into account the definition of the set $M\left(j_{0}\right)$, we conclude that $k_{0} \notin M\left(j_{0}\right)$. However, this contradicts the assumption that $k_{0} \in M\left(j_{0}\right)$. The resulting contradiction proves that equalities (A9) hold true.

Now we are ready to prove Lemma 2. In fact, under the assumption $X \neq \varnothing$, from Propositions 2 and 6 it follows that

$$
T_{i m}(X)=T_{i m}(\mathcal{Z}) \text { and }\left\{\tau^{*}(j), j \in J\right\} \subset\left\{\tau(i), i \in I_{m_{*}}\right\} \subset T_{i m}(\mathcal{Z}) .
$$

Hence, $\left\{\tau(i), i \in I_{m_{*}}\right\} \subset \operatorname{conv}\left\{\tau^{*}(j), j \in J\right\}$. Using this inclusion, it is easy to show that $Z_{*}=Z\left(m_{*}\right)$. From the latest equality, the inclusion $\left\{\tau^{*}(j), j \in J\right\} \subset\left\{\tau(i), i \in I_{m_{*}}\right\}$, and the definitions of the sets $\mathcal{F}_{m_{*}}^{3}$ and $F_{*}$ (see (39) and (A7)), it follows that $\mathcal{F}_{m_{*}}^{3} \subset F_{*}$ and both faces, $F_{*}$ and $\mathcal{F}_{m_{*}}^{3}$, contain the set $\mathcal{D}$.

On the other hand, Proposition A1 states that $F_{*}$ is the minimal face containing the set $\mathcal{D}$. This implies that $\mathcal{F}_{m_{*}}^{3}=F_{*}=\mathbf{F}_{\text {min }}$. Lemma 2 is proved.

\section{References}

1. Bomze, I.M. Copositive optimization -recent developments and applications. EJOR 2012, 216, 509-520. [CrossRef]

2. de Klerk, E.; Pasechnik, D.V. Approximation of the stability number number of a graph via copositive programming. SIAM J. Optim. 2002, 12, 875-892. [CrossRef]

3. Dür, M. Copositive Programming-A survey. In Recent Advances in Optimization and Its Applications in Engineering; Diehl, M., Glineur, F., Jarlebring, E., Michielis, W., Eds.; Springer: Berlin/Heidelberg, Germany, 2010; pp. 3-20.

4. Letchford, A.N.; Parkes, A.J. A guide to conic optimisation and its applications. RAIRO-Rech. Oper. Res. 2018, 52, 1087-1106. [CrossRef]

5. Ahmed, F.; Dür, M.; Still, G. Copositive Programming via semi-infinite optimization. J. Optim. Theory Appl. 2013, 159, 322-340. [CrossRef]

6. Anjos, M.F.; Lasserre, J.B. (Eds.) Handbook of Semidefinite, Conic and Polynomial Optimization; International Series in Operational Research and Management Science; Springer: Berlin/Heidelberg, Germany, 2012; Volume 166, 138p.

7. Wolkowicz, H.; Saigal, R.; Vandenberghe, L. (Eds.) Handbook of Semidefinite Programming: Theory, Algorithms, and Applications; Kluwer Academic Publishers: Berlin/Heidelberg, Germany, 2000.

8. Kortanek, K.O.; Zhang, Q. Perfect duality in semi-infinite and semidefinite programming. Math. Program. Ser. A 2001, 91, 127-144. [CrossRef]

9. Borwein, J.M.; Wolkowicz, H. Facial reduction for a cone-convex prograing problem. J. Austral. Math. Soc. Ser. A 1981, 30, 369-380. [CrossRef]

10. Borwein, J.M.; Wolkowicz, H. Regularizing the abstract convex program. J. Math. Anal. Appl. 1981, 83, 495-530. [CrossRef]

11. Luo, Z.-Q.; Sturm, F.J.; Zhang, S. Duality Results for Conic Convex Programming; Econometric Institute Report no. 9719/a; Erasmus University Rotterdam, Erasmus School of Economics (ESE), Econometric Institute: Rotterdam, The Netherlands , 1997. 
12. Waki, H.; Muramatsu, M. Facial reduction algorithms for conic optimization problems. J. Optim. Theory Appl. 2013, 158, 188-215. [CrossRef]

13. Pataki, G. A Simple Derivation of a Facial Reduction Algorithm and Extended Dual Systems. 2000. Available online: https: //gaborpataki.web.unc.edu/wp-content/uploads/sites/14119/2018/07/fr.pdf (accessed on 6 February 2022).

14. Permenter, F.; Friberg, H.; Andersen, E. Solving conic optimization problems via self-dual embedding and facial reduction: A unified approach. SIAM J. Optim. 2015, 27, 1257-1282. [CrossRef]

15. Pólik, I.; Terlaky, T. Exact Duality for Optimization over Symmetric Cones; AdvOL-Report No. 2007/10; McMaster University, Advanced Optimization Lab.: Hamilton, ON, Canada, 2007.

16. Ramana, M.V.; Tunçel, L.; Wolkowicz, H. Strong duality for Semidefinite Programming. SIAM J. Optim. 1997, 7, 641-662. [CrossRef]

17. Kostyukova, O.I.; Tchemisova, T.V. Face reduction and the immobile indices approaches to regularization of linear Copositive Programming problems. arXiv 2021, arXiv:2109.00080.

18. Kostyukova, O.I.; Tchemisova, T.V. On equivalent representations and properties of faces of the cone of copositive matrices. arXiv 2020, arXiv:2012.03610v1.

19. Kostyukova, O.I.; Tchemisova, T.V.; Dudina, O.S. Immobile indices and CQ-free optimality criteria for linear copositive programming problems. Set-Valued Var. Anal. 2020, 28, 89-107. [CrossRef]

20. Kostyukova, O.; Tchemisova, T. On strong duality in linear copositive programming. J. Glob. Optim. 2021. 1-24. [CrossRef]

21. Bonnans, J.F.; Shapiro, A. Perturbation Analysis of Optimization Problems; Springer: Berlin/Heidelberg, Germany, 2000; 601p.

22. Levin, V.L. Application of E. Helly's theorem to convex programming, problems of best approximation and related questions. Math. USSR Sbornik 1969, 8, 235-247. [CrossRef]

23. Drusvyatskiy, D.; Wolkowicz, H. The Many Faces of Degeneracy in Conic Optimization. Foundations and Trends in Optimization; Now Publishers Inc.: Delft, The Netherlands: 2017; Volume 3, pp. 77-170.

24. Hettich, R.; Kortanek, K.O. Semi-infinite programming: Theory, methods, and applications. SIAM Rev. 1993, 35, 380-429. [CrossRef]

25. Tunçel, L.; Wolkowicz, H. Strong duality and minimal representations for cone optimization. Comput. Optim. Appl. 2012, 53, 619-648. [CrossRef] 